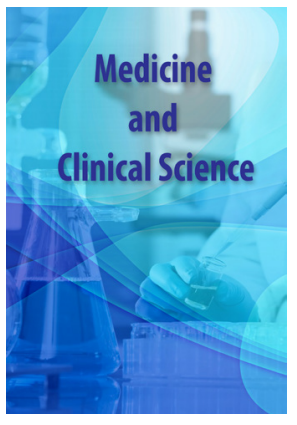

Correspondence

Louise Fish

Department of General Medicine, Bunbury Regional Hospital, Australia

E-mail: louise.fish@health.wa.gov.au

- Received Date: 05 Nov 2019;

- Accepted Date: 23 Nov 2019,

- Publication Date: 17 Dec 2019.

Copyright

(C) 2020 Science Excel. This is an openaccess article distributed under the term of the Creative Commons Attribution 4.0 International license.

\title{
Case report - meningitic symptoms in immunocompetent patient: an unusual case of cryptococcus gatti infection
}

\author{
Fish L, Ekladious A and Wheeler LP \\ Department of General Medicine, Bunbury Regional Hospital, Australia
}

\section{Case}

A 25 year-old male presented to a rural hospital emergency department with a seven day history of headaches, photophobia, myalgia, fevers, vomiting and unusual behaviour. The patient was previously fit and well with no significant past medical history, but reported daily marijuana use. Physical examination revealed mild neck stiffness, but no headache, rash or photophobia. The patient was admitted under the general medical team for investigation and management of potential meningitis or drug-induced psychosis.

Imaging including computed tomography (CT) brain was unremarkable and blood panels showed a mildly elevated CRP of $44 \quad(<3)$ and normal white cell count of $6000 / \mu \mathrm{L}(4000-11000 / \mu \mathrm{L})$ with polymorphonuclear leucocytosis.. A lumbar puncture was performed and cerebrospinal fluid (CSF) analysis showed no bacteria on gram stain or growth on culture, marginally elevated glucose $3.7 \mathrm{mmol} / \mathrm{L} \quad(2.5-3.5 \mathrm{mmol} / \mathrm{L})$, elevated protein $717 \mathrm{mg} / \mathrm{L} \quad(180-450 \mathrm{mg} / \mathrm{L})$, leucocytes $50 / \mu \mathrm{L}$, neutrophils $30 / \mu \mathrm{L}$ (white cells $<5 / \mu \mathrm{L}$ ) With nonspecific findings, dexamethasone, ceftriaxone and acyclovir were commenced to cover bacterial and viral meningitis.

After discussion with Infectious Disease physicians, he was diagnosed with bacterial meningitis and was discharged with intravenous ceftriaxone for fourteen days.

Seven days later, he was reviewed in the outpatient clinic and had persistent headaches, photophobia and neck pain and was re-admitted under the general medical team. Lumbar puncture was repeated and CSF showed elevated white cells (lymphocytes $20 / \mu \mathrm{L}$ neutrophils $10 / \mu \mathrm{L}$ ), normal glucose $2.7 \mathrm{mmol} / \mathrm{L}$, and was positive on India Ink testing. Fungal cultures were conducted on this occasion and growth of Cryptococcus gatti was identified,. Subsequently magnetic resonance imaging (MRI) of the brain revealed multiple foci of signal abnormalities, subacute infarcts and parenchymal and leptomningeal enhancement., $\mathrm{He}$ was diagnosed with cryptococcal meningitis, treated with Amphotericin B and up-transferred to a tertiary center.

During this admission, a third LP was performed. Opening pressure was measured on this occasion, and found to be elevated at $40 \mathrm{mmHg}$ (normal range $8-15 \mathrm{mmHg}$ ) requiring subsequent insertion of a ventriculoperitoneal (VP) shunt. Intravenous flucytosine was then prescribed with Amphotericin $B$ and continued for 6 weeks, stepped down to oral fluconazole $800 \mathrm{mg}$ for eight weeks, then $400 \mathrm{mg}$ for six months.
Cryptococcal meningitis is an opportunistic fungal infection caused by inhaling spores of Cryptococcus neoformans yeast [1]. C. gattii is the primary serotype in the immunocompetent patient population and infections tend to present as afebrile, with shorter symptom duration, and focal pulmonary and central nervous system mass lesions [2,3]. Diagnosis made with positive serum or CSF culture, but as seen in this case, repeat lumbar puncture with large samples may be required to mitigate high false negatives rates $[4,5]$.

With multiple non-specific signs and symptoms, this case highlights the importance of considering all differential diagnoses, and being aware of so called 'red herrings'. Clinicians should seek alternative diagnoses when routine treatment is ineffective. In this case, differential diagnoses should have been considered when the standard antibiotic steroid therapy regimen showed little response.

This case also highlights the importance of performing opening pressures during the lumbar puncture procedure. Specifically for cryptococcal meningitis, raised intracranial pressure is a diagnostic characteristic in $50-70 \%$ of patients. Identification of raised ICP early can prevent delayed diagnosis and treatment as well as prevent procedure repetition which is both time consuming for the clinician, and uncomfortable for the patient and had this been addressed on initial admission, prompt transfer to a tertiary center would have occurred and appropriate treatment commenced. Crucially, the VP shunt may have been avoided, as delayed diagnosis led to complications from raised intracranial pressure.

\section{References}

1. Chang CC, Perfect JR. Repeated therapeutic lumbar punctures in cryptococcal meningitis-necessity and/or opportunity? Current opinion in infectious diseases. 2016;29(6):539-45

2. Bicanic T, Harrison TS. Cryptococcal meningitis. British medical bulletin. 2004;72(1):99-118.

3. Ming DK, Harrison TS. Cryptococcal meningitis. British Journal of Hospital Medicine. 2017;78(8):C125-C7.

4. Berlin L, Pincus JH. Cryptococcal meningitis: false-negative antigen test results and cultures in nonimmunosuppressed patients. Archives of neurology. 1989;46(12):1312-6.

5. Wilson A, Wilkie $M$, Rae N. Fungal diseases at the medical front door. British Journal of Hospital Medicine. 2019;80(3):157-61.
Citation: Fish L. Case report - meningitic symptoms in immunocompetent patient: an unusual case of cryptococcus gatti infection. Med Clin Sci. 2020;2(1):1-10. 